\title{
EDITORS' CHOICE
}

\section{The importance of primary care research in the management of respiratory disease}

- We are delighted to start the year with an important paper from the IPCRG (Pinnock et al., pg 19) which, together with the linked editorial by Holgate (pg 1), both highlight the importance of primary care research in respiratory medicine and the need for it be funded appropriately. Pinnock et al. have prioritised the original 145 research questions in the earlier IPCRG Research Needs Statement (http://dx.doi.org/10.4104/pcrj.2010.00021), using a three-stage e-Delphi process involving 23 international experts. 62 research questions obtained the $80 \%$ priority threshold and seven achieved $100 \%$ consensus. Holgate emphasises the significance of this paper and, using the example of the 3-fold increase in respiratory research funding which followed the formation of the multi-professional UK Respiratory Research Collaborative (UKRRC), demonstrates how the respiratory research community can work together to meet these exciting challenges.

\section{Statins for COPD: a challenge to conventional beliefs?}

- On pg 35, Lawes et al. present the first national cohort study to assess whether statin use is associated with reduced mortality in COPD patients. Using hospitalisation, drug dispensing and mortality records for all New Zealanders aged 50-80 discharged from hospital with a first admission for COPD, 596 statin users and 1,091 non-users were followed-up for up to 4 years. After adjustment for age, sex, ethnic group, history of heart disease, diabetes and prescriptions for frusemide, statin use was associated with a 30\% reduction in all-cause mortality at 3-4 years, irrespective of a past history of heart disease or diabetes. In his thought-provoking editorial on pg 5, Pearson postulates what this might mean, and calls for an urgent RCT on statin use in COPD.

\section{SMART in primary care: where are we?}

- Riemersma et al. (pg 50) report a study on 102 primary care patients with mild-to-moderate asthma who received either budesonide/formoterol as Single inhaler Maintenance And Reliever Therapy (SMART) or usual care treatment, in which the primary outcome was bronchial hyper-reactivity (BHR) measured by PD20-histamine at 12 months. Despite a 59\% lower dose of ICS, BHR and other outcomes remained stable in the SMART group and peak flow values improved. In their linked editorial (pg 8), Thomas and Pavord summarise the ongoing controversy over SMART and put this paper in context.

\section{Asthma deaths - identifying risk factors early}

- The UK National Review of Asthma Deaths started on 1st February 2012 (see correspondence from Levy on pg 18), so the paper by Anagnostou et al. (pg 71) is highly topical. The authors report on paediatric deaths occurring over 2001-2006 in the Eastern Region of the UK. 20 children (median age 11.5 years) died, nine of whom had presumed mild-moderate asthma. Half died in the summer months, suggesting a seasonal cause, but only three had had formal allergy testing. Six children had been prescribed long-acting $\beta 2$-agonist (LABA) inhalers but were not on inhaled steroids. Stephenson and Shields put this paper in context (pg 13), and call for the construction of primary care at-risk asthma registers.

\section{Dawning of a new lung age?}

- The 2010 PCRJ paper by Newbury et al., (http://dx.doi.org/10.4104/pcrj.2010.00029) on the need to update lung age equations, is now followed by their paper on $\mathrm{pg} 78$, in which they present new lung age equations which perform better than those used previously. In his linked editorial (pg 15), Cooper congratulates the authors on a well-designed study, but concludes that the intrinsic limitations of using 'lung age' mean that the concept should not be adopted widely and that this is another 'false dawn' for lung age. What do you think? We will be delighted to receive correspondence on this important issue.

\section{Journalwatch@pcrj: a new section in the PCRJ}

- Finally, on pg 115, we present a new section in the PCRJ, Journalwatch@pcrj. This will be a regular feature in each issue from now on, containing summary reviews of relevant papers from the top respiratory and general medical journals worldwide. We hope you find these useful!

\section{Paul Stephenson and Aziz Sheikh}

